\title{
EFICIENCIA A FÓSFORO EN GERMOPLASMA DE MAÍZ DE LA MESETA P’URHÉPECHA EN ETAPA DE PLÁNTULA
}

\author{
PHOSPHORUS-EFFICIENCY IN MAIZE GERMPLASM AT SEEDLING \\ STAGE FROM THE P'URHÉPECHA PLATEAU
}

\author{
Jeannette S. Bayuelo-Jiménez ${ }^{1 *}$, Iván Ochoa ${ }^{2}$, Verónica A. Pérez-Decelis ${ }^{1}$, Ma. de Loudes \\ Magdaleno-Armas ${ }^{1}$ y Raúl Cárdenas-Navarro ${ }^{1}$
}

\begin{abstract}
${ }^{1}$ Instituto de Investigaciones Agropecuarias y Forestales, Universidad Michoacana de San Nicolás de Hidalgo. Km 9.5 Carr. Morelia - Zinapécuaro. 58880, Tarímbaro, Michoacán, México. Tel. y Fax 01(443) 2958324. ${ }^{2}$ Unipalma S.A. Calle 74 A No. 22-31. Bogotá, Colombia.

*Autor para correspondencia (bayuelo@umich.mx)
\end{abstract}

\section{RESUMEN}

La reducida disponibilidad de fósforo $(\mathrm{P})$ en el suelo es uno de los principales factores que limita la producción de maíz (Zea mays L.), particularmente en suelos ácidos. Los cambios en la morfología y arquitectura radical están regulados por la disponibilidad de $P$ en el suelo y son importantes para su adquisición. Se estudió la variabilidad genotípica de maíz de la Meseta P’urhépecha, en Michoacán, México, para eficiencia a fósforo y las características radicales asociadas con dicha eficiencia, en etapa de plántula. El experimento se efectuó en condiciones de invernadero e hidroponía, con dos niveles de disponibilidad de fósforo $\left(\mathrm{KH}_{2} \mathrm{PO}_{4}\right)$, baja $(1 \mu \mathrm{M})$ y alta $(1 \mathrm{mM})$. A los $7 \mathrm{~d}$ se analizó el crecimiento de la plántula, la morfología y arquitectura radical. Los genotipos de maíz difirieron en crecimiento vegetal, morfología radical y eficiencia a fósforo, definida esta última como el crecimiento sostenido en condiciones de baja disponibilidad de fósforo. Los genotipos se agruparon en tres categorías de eficiencia con base en sus parámetros de crecimiento en el nivel bajo en fósforo y sus valores relativos al tratamiento de alto fósforo, y en cuatro categorías de acuerdo con el valor de eficiencia (IEF) en combinación con la respuesta en crecimiento en el nivel alto de fósforo. Los genotipos eficientes tuvieron mayor peso seco de raíz y de vástago, mayor asignación de materia seca a la raíz, raíces seminales de mayor longitud y pelos radicales más densos en la raíz seminal y primaria; en este grupo quedaron incluidas variedades nativas (criollos) y criollos mejorados. Estos resultados indican que en el germoplasma de maíz de la Meseta P'urhépecha existe variabilidad genotípica en la eficiencia a fósforo y la arquitectura radical.

Palabras clave: Zea mays, crecimiento, arquitectura radical, eficiencia a fósforo, suelos ácidos.

\section{SUMMARY}

Reduced phosphorus (P) availability in the soil is one of the main yieldlimiting factors for maize (Zea mays L.) production, particularly on acid soils. Changes in root morphology and architecture are regulated by $P$ availability and are particularly important for phosphorus acquisition. This study was conducted to evaluate the genotypic variability of maize from the P'urhépecha Plateau in Michoacán, México, by quantifying $P$ efficiency and root characteristics associated with $P$ efficiency at seedling stage. The experiment was conducted under greenhouse conditions, in nutrient solution at low $(1 \mu \mathrm{M})$ and high $(1 \mathrm{mM}) \mathrm{KH}-$ ${ }_{2} \mathrm{PO}_{4}$ availability. Plant growth traits, root architecture and morphology were evaluated for $7 \mathrm{~d}$. Accessions differed greatly in plant growth, root morphology and $\mathbf{P}$ efficiency (defined as growth under suboptimal $\mathbf{P}$ availability). The accessions were divided into three categories based on $P$ efficiency and four categories according to $P$ efficiency in combination with high phosphorus response. P-efficient accessions had greater values of root and shoot dry matter production, root to shoot ratio, seminal root length, and root hairs density of seminal and primary roots.
The increase in root hair density and in seminal root length resulted in increased plant growth under low $\mathrm{P}$, at seedling stage. These results indicate that maize from the P'urhépecha Plateau exhibit variation for $P$ efficiency and root architecture.

Index words: Zea mays, plant growth, root architecture, $\mathrm{P}$ efficiency, acid soils.

\section{INTRODUCCIÓN}

La reducida disponibilidad de fósforo $(\mathrm{P})$ en el suelo es uno de los principales factores que limita el crecimiento y rendimiento de los cultivos a nivel mundial, particularmente en los países en donde el acceso al fertilizante es restringido (Lynch, 2007). El mejoramiento genético conducente al desarrollo de genotipos eficientes que mantengan o incrementen el crecimiento y rendimiento en condiciones de baja disponibilidad de $\mathrm{P}$ en el suelo, es una de las estrategias biológicas más atractivas para aumentar la productividad (Richardson et al., 2011).

El fósforo es relativamente inmóvil en el suelo y su disponibilidad decrece con la profundidad (Lynch, 2007). Las raíces adquieren el $\mathrm{P}$ de la solución del suelo en forma de fosfato $\left(\mathrm{P}_{\mathrm{i}}\right)$, primariamente en la forma de $\mathrm{KH}_{2} \mathrm{PO}_{4}$ (Vance et al., 2003). Sin embargo, la concentración en la solución del suelo es reducida $(0.1$ a $10 \mu \mathrm{M})$ y el acceso de $\mathrm{P}_{\mathrm{i}}$ hacia la superficie de las raíces por difusión es limitado (Hinsinger, 2001). Las plantas han desarrollado diferentes estrategias fisiológicas y morfológicas para adaptarse a condiciones de escasez de $\mathrm{P}$ disponible (Vance et al., 2003). Las características de la arquitectura radical asociadas directamente con el incremento de la adquisición de $\mathrm{P}$ en las capas superficiales del suelo incluyen la formación de raíces basales superficiales (Bonser et al., 1996), la producción de raíces adventicias (Miller et al., 2003; Ochoa et al., 2006), la formación y dispersión de raíces laterales (Zhu y Lynch, 2004) y mayor longitud o densidad de pelos radicales (Jungk, 2001). 
La morfología y la configuración de las raíces en espacio y tiempo (arquitectura radical) se han utilizado en programas de mejoramiento de maíz (Zea mays L.), particularmente en áreas con bajos niveles de $\mathrm{P}$ disponible en el suelo (Lynch, 2007). La investigación en este ámbito por lo general se ha realizado con variedades mejoradas o líneas isogénicas derivadas de un limitado acervo de germoplasma y predominantemente adaptadas a una agricultura intensiva y ambientes óptimos (Kochian et al., 2005).

En contraste, las variedades tradicionales de maíz se desarrollan en ambientes específicos o marginados con baja disponibilidad de nutrientes por lo que representan una fuente importante de variación para la selección de variedades adaptadas a los agrosistemas rurales (Newton et al., 2010). Las variedades tradicionales de maíz de la Subprovincia Neovolcánica P'urhépecha están adaptadas a las condiciones agroecológicas de la región y es posible que dispongan de caracteres radicales que le confieran a la especie una mayor eficiencia a fósforo. Esta región se caracteriza por una gran diversidad de microclimas con altitudes que oscilan de 1000 a $3800 \mathrm{~m}$ y un clima subhúmedo $(\mathrm{Cw})$, cuyo suelo es de tipo Andosol vítrico con alta capacidad de intercambio catiónico (12 a $\left.75 \mathrm{cmol} \mathrm{kg}^{-1}\right)$, baja saturación de bases $(<50$ $\%), \mathrm{pH}$ ácido (4.9 a 5.58) y alta fijación de fosfatos (> $75 \%$ ) (Alcalá et al., 2001).

En la selección de genotipos eficientes a fósforo se han propuesto diferentes índices de eficiencia, los que incluyen una sola característica de la planta o varias (Li et al., 2003). La selección de genotipos basados en un solo carácter de eficiencia puede involucrar la comparación directa del peso seco del vástago, el incremento de la relación raíz:vástago, la cantidad de fósforo absorbido (Fageria et al., 2008) o la arquitectura radical (Lynch, 2007). En la selección de genotipos eficientes basados en parámetros múltiples es posible integrar los efectos de los diferentes caracteres mediante métodos de clasificación (Li et al., 2003). Sin embargo, estos métodos omiten la contribución relativa de los diferentes parámetros para estimar la eficiencia a fósforo como lo hace un análisis de componentes principales (Pan et al., 2008). Estudios de selección de eficiencia en soya (Glycine max L.)
(Pan et al., 2008) y maíz (Bayuelo-Jiménez et al., 2011) demuestran la conveniencia del uso de ambos métodos.

El objetivo de este estudio fue evaluar la variabilidad genotípica de maíz de la Meseta P'urhépecha para eficiencia a fósforo y las características radicales asociadas con dicha eficiencia, en etapa de plántula.

\section{MATERIALES Y MÉTODOS}

El experimento incluyó dos niveles de fósforo, baja $(1 \mu \mathrm{M}$; BP) y alta disponibilidad ( $1 \mathrm{mM}$; $\mathrm{AP}$ ) de $\mathrm{KH}_{2} \mathrm{PO}_{4}$, combinados con 108 genotipos de maíz provenientes del programa de mejoramiento del Instituto Nacional de Investigaciones Forestales, Agrícolas y Pecuarias, integrados por criollos de la Meseta P'urhépecha, criollos cruzados con maíces mejorados, híbridos cruzados con criollos y variedades sintéticas formadas por estos mismos materiales. Los materiales se clasificaron como poblaciones de amplia base genética de acuerdo con el número de d para alcanzar 50 \% de floración masculina, en: precoces ( 75 a $80 \mathrm{~d}$ con 33 materiales), intermedios ( 85 a 95 d con 40 materiales) y tardíos ( 95 a 110 d con 35 materiales) y cuatro grados de mejoramiento: criollos (C), criollos mejorados $(\mathrm{CM})$, híbridos x criollo $(\mathrm{H} \times \mathrm{C})$ e híbridos mejorados (S), como se detalla en el Cuadro 1.

Antes de establecer el experimento, las semillas se esterilizaron con hipoclorito de sodio a $5 \%(\mathrm{v} / \mathrm{v})$ y agua demonizada. En cada unidad experimental se seleccionaron ocho semillas por genotipo y se colocaron a $2 \mathrm{~cm}$ del borde de la hoja del papel de germinación (Anchor Paper Co., St. Paul, $\mathrm{MN}$, USA), se enrollaron y colocaron en oscuridad en una

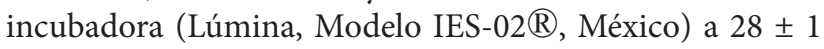
${ }^{\circ} \mathrm{C}$ y humedad relativa de $65 \%$, por $3 \mathrm{~d}$. Una vez germinadas, cuatro plántulas se colocaron en recipientes aireados $(4$ $\mathrm{L}$ de capacidad) con solución nutritiva [en $\mu \mathrm{M}$ : 3000 (K), $7000\left(\mathrm{NO}_{3}\right), 1000\left(\mathrm{NH}_{4}\right), 2000(\mathrm{Ca}), 500\left(\mathrm{SO}_{4}\right), 500(\mathrm{Mg})$, $25(\mathrm{Cl}), 12.5(\mathrm{~B}), 1.0(\mathrm{Mn}), 1.0(\mathrm{Zn}), 0.25(\mathrm{Cu}), 0.25(\mathrm{Mo})$, y 25 (EDTA-Fe) ] con bajo $(1 \mu \mathrm{M})$ y alto $(1 \mathrm{mM})$ contenido de fósforo. Las plántulas se desarrollaron en una cámara de crecimiento a una temperatura de $28 \pm 24{ }^{\circ} \mathrm{C}$ (luz/oscuridad) a una intensidad luminosa de $200 \mu \mathrm{mol}$ fotones

Cuadro 1. Clasificación de los 108 genotipos de maíz en etapa de plántula.

\begin{tabular}{lccc}
\hline \multirow{2}{*}{ Tipos de maíz } & \multicolumn{3}{c}{ Grupos de madurez } \\
\cline { 2 - 4 } & $\begin{array}{c}\text { I (Precoces) } \\
(33)\end{array}$ & $\begin{array}{c}\text { II (Intermedios) } \\
(40)\end{array}$ & $\begin{array}{c}\text { III ( Tardíos) } \\
(35)\end{array}$ \\
\hline Criollos (C) & 9 & 19 & 22 \\
Criollos mejorados (CM) & 10 & 11 & 9 \\
Híbridos x Criollos (H x C) & 8 & 7 & 2 \\
Sintéticos (S) & 6 & $z 3$ & 2 \\
Días a floración masculina & $75-85$ & $85-95$ & $95-110$ \\
\hline
\end{tabular}


$\mathrm{m}^{-2} \mathrm{~s}^{-1} \mathrm{y}$ humedad relativa de $65 \%$. La solución nutritiva se mantuvo a un $\mathrm{pH}$ de 6.0 con una solución amortiguadora de $\mathrm{KOH} 1 \mathrm{~N}$.

Las plántulas se cosecharon $7 \mathrm{~d}$ después de haberse desarrollado en los tratamientos de alto y bajo P. En ese momento, el sistema radical del maíz consistía de una raíz primaria con raíces laterales y de dos a seis raíces seminales, según la descripción de Hochholdinger et al. (2004). En las muestras colectadas de raíz se evaluaron cinco características: longitud de la raíz primaria, longitud y número de raíces seminales, y densidad y longitud de los pelos radicales. Las raíces se almacenaron en etanol a $25 \%(\mathrm{v} / \mathrm{v})$ hasta su tinción con azul de toluidina $0.5 \%$ para la evaluación de los pelos radicales. La densidad y la longitud de los pelos radicales de la región basal de la raíz primaria y de las raíces seminales se evaluaron visualmente con la escala: $1=$ sin pelos radicales; 3 = baja densidad/longitud; 5 a 7 = intermedia densidad/ longitud; 9 = abundante densidad/longitud de pelos radicales (Vieira et al., 2007). Se utilizó un microscopio estereoscópico (Zeiss STEMI DV4®; Göttingen, Germany) con un aumento de 30X. Las hojas y raíz de cada conjunto de cuatro plántulas/genotipo se deshidrataron en una estufa a 65 ${ }^{\circ} \mathrm{C}$ por $48 \mathrm{~h}$, para luego medir el peso seco. Se cuantificó la concentración de fósforo en la semilla de los 108 genotipos de maíz mediante el método colorimétrico propuesto por Murphy y Riley (1962).

Para el análisis estadístico de los datos se empleó un diseño de bloques al azar con arreglo de tratamientos de parcelas divididas y cinco repeticiones por tratamiento. Los niveles de fósforo correspondieron a la parcela principal, y los genotipos distribuidos aleatoriamente dentro de cada parcela correspondieron a las subparcelas. La eficiencia a fósforo, definida como el crecimiento sostenido en condiciones de baja disponibilidad de este elemento, se determinó con el índice de eficiencia a fósforo (IEF) propuesto por Pan et al. (2008) el cual se obtiene mediante un análisis de componentes principales (ACP) a partir de datos estandarizados del peso seco de la raíz, del vástago, peso seco total y la relación raíz:vástago (RRV) en condiciones de BP y relativo a la condición en AP, con el paquete de Statistical Analysis System (SAS Institute, 2000).

Los valores propios del ACP $\geq 1$ se retuvieron para el cálculo del IEF. Los valores del IEF de cada genotipo se calcularon de acuerdo con el componente principal retenido (CP) y su contribución relativa (CR): IEF $={ }^{108} \sum_{\mathrm{i}=1} C P_{i}$ $\mathrm{x} \mathrm{CR}_{\mathrm{i}}$. Posteriormente, con los datos de IEF por genotipo se hizo un análisis de conglomerados para agrupar por categorías de eficiencia [eficientes (E), moderadamente eficientes (ME) e ineficientes (I)] y cuatro categorías de respuesta a $\mathrm{P}$ de acuerdo con el IEF en combinación con la respuesta a $\mathrm{P}$ (peso seco del vástago en alto fósforo) [efi- cientes y con respuesta (ER), no eficientes y con respuesta (NER), no eficientes y sin respuesta (NENR), y eficientes y sin respuesta (ENR)].

El análisis de conglomerados se hizo con el método de Ward; el número óptimo de grupos de acuerdo con el IEF se determinó por el índice de suma de cuadrados (Romerburg, 1984). Posteriormente, se ubicaron los genotipos en un plano cartesiano de acuerdo con el IEF y con la respuesta a $\mathrm{P}$ (peso seco del vástago en alto fósforo, estandarizado). Finalmente, se realizó un análisis de correlación entre el índice de eficiencia a fósforo y las características de la arquitectura radical (SAS Institute, 2000).

\section{RESULTADOS}

\section{Eficiencia a fósforo}

Según el análisis de componentes principales (CP) de las características de crecimiento de los genotipos evaluados, los componentes CP1 al CP3 contribuyeron con 39, 31 y $22 \%$ de la variación entre grupos. Estos componentes en conjunto aportaron $92 \%$ de la variación total observada. La variabilidad del primer componente (CP1) estuvo determinada por valores positivos de peso seco de la raíz (0.89), peso seco de vástago (0.92) y peso seco total (0.99) en BP. El CP2 estuvo influenciado positivamente por la relación raíz:vástago (RRV; 0.86) en condiciones de $\mathrm{BP}$, y los valores relativos a la condición en AP del peso seco de la raíz (0.69) y RRV (0.96). En el CP3 ejercieron mayor peso los valores relativos del peso seco de raíz (0.66), peso seco de vástago $(0.88)$ y peso seco total $(0.98)$.

En la Figura 1 se presenta la formación de los grupos de eficiencia (IEF) de acuerdo con un análisis de conglomerados: El Grupo I (IEF < -0.845) consistió de seis genotipos ineficientes, de los cuales 66.7 y $33.3 \%$ fueron de madurez intermedia y tardía. El Grupo II $(-0.845<$ IEF $<-0.104)$ estuvo compuesto por 53 genotipos moderadamente eficientes, con $32,37.7$ y $30 \%$ de madurez precoz, intermedia y tardía. El Grupo III (IEF > 0.216) lo integraron 49 genotipos eficientes a fósforo, la mayoría de los cuales fueron variedades criollas (39\%) y criollas mejoradas $(20.4 \%)$ de madurez intermedia (32.6\%) y tardía (34.7\%).

Los grupos de eficiencia difirieron en el patrón de crecimiento y arquitectura radical (Cuadro 2). El peso seco acumulado en raíz y vástago y la RRV incrementó en los grupos de mayor eficiencia en condiciones de BP. En lo que refiere a la arquitectura radical, la longitud de la raíz primaria y de las raíces seminales aumentó en los genotipos de moderada y alta eficiencia, aunque la respuesta fue similar en AP y BP. La longitud y la densidad de los pelos radicales de la raíz seminal incrementó en BP, pero la respuesta fue 


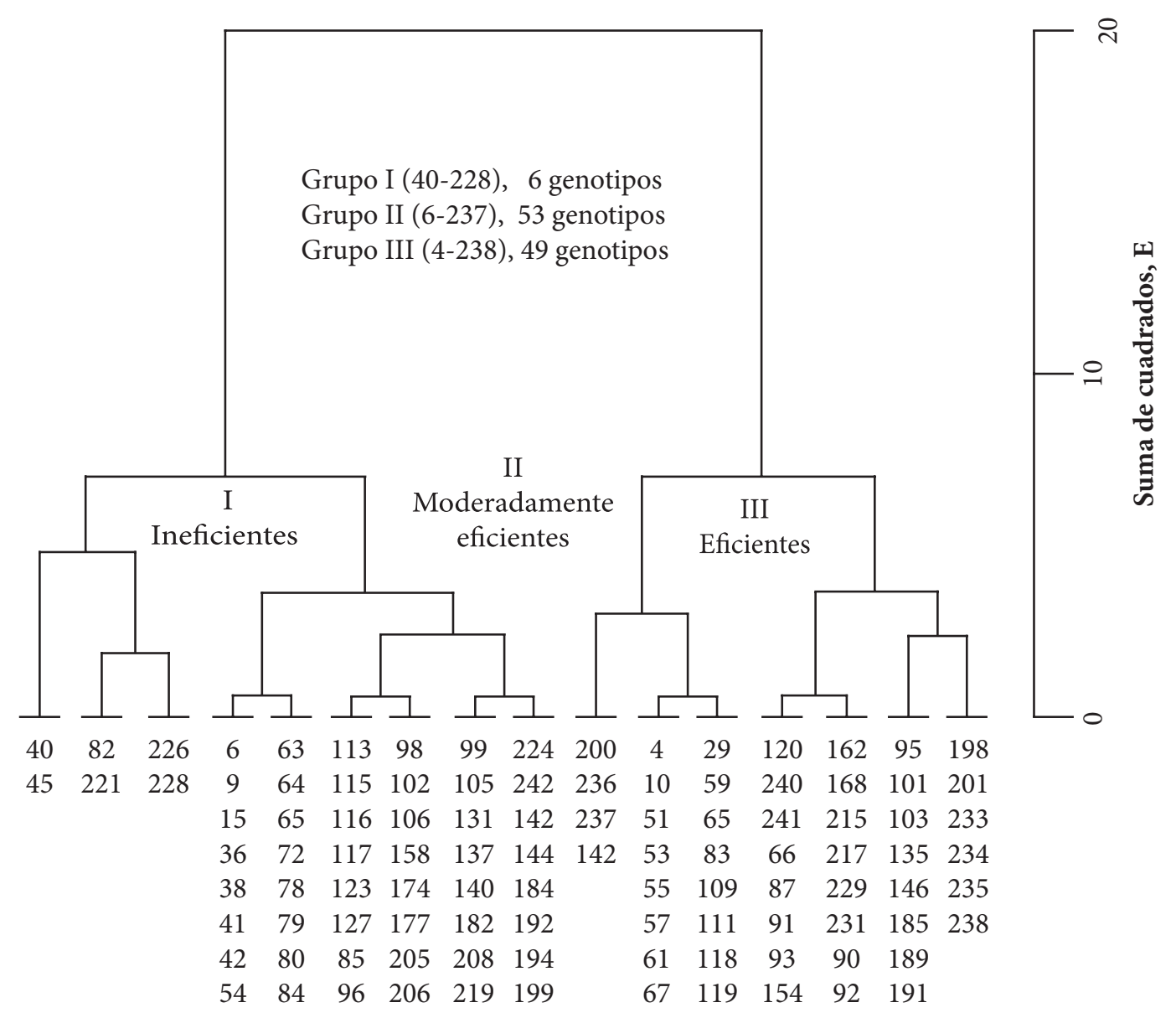

Figura 1 Clasificación de 108 genotipos de maíz de acuerdo con el índice de eficiencia a fósforo (IEF), mediante el metodo de agrupación de Ward.

independiente de los grupos de eficiencia. En contraste, la raíz primaria desarrolló pelos radicales más densos o más largos en los genotipos más eficientes.

\section{Eficiencia y respuesta a fósforo}

Los 108 genotipos se dividieron en cuatro grupos de acuerdo con su respuesta a fósforo (expresada por el peso seco del vástago en alto fósforo, estandarizado) y eficiencia a fósforo (IEF). Las categorías fueron: (i) con eficiencia y respuesta (ER), (ii) sin eficiencia y con respuesta (NER), (iii) sin eficiencia y respuesta (NENR) y (iv) con eficiencia y sin respuesta (ENR), como se muestra en la Figura 2 y en el Cuadro 3.

En la comparación de la respuesta y eficiencia a fósforo se identificaron 34 genotipos eficientes en $\mathrm{BP}$ y que respondieron a la aplicación del mismo (Cuadro 3). Estos genotipos, particularmente 14 criollos y 11 criollos mejorados, exhibieron una similar acumulación del peso seco del vás- tago (76.4 y $75.3 \mathrm{mg}$ ) en ambos ambientes. Veinticinco genotipos presentaron una alta respuesta a $\mathrm{P}$, manifestada por un incremento en el peso seco del vástago $(73.6 \mathrm{mg} /$ planta $)$ pero fueron ineficientes en la acumulación de materia seca (68.7 mg/planta) en BP; mientras que 28 genotipos fueron ineficientes a BP y no respondieron a la condición de AP. En la categoría ENR se ubicaron 21 genotipos de moderada y alta eficiencia, particularmente 13 variedades criollas que incrementaron el peso seco del vástago (>70 mg/planta) en el ambiente de BP pero no respondieron a la mayor disponibilidad del mismo (Cuadro 3).

\section{Correlación entre peso seco del vástago y arquitectura radical}

Las correlaciones entre niveles de fósforo fueron positivas y significativas para longitud de la raíz primaria $(\mathrm{r}$ $=0.56)$, la longitud de la raíz seminal $(\mathrm{r}=0.67)$, longitud de los pelos radicales en la región basal de la raíz seminal $(\mathrm{r}=0.66)$ y peso seco del vástago $(\mathrm{r}=0.51)$. Las mayores 
Cuadro 2. Parámetros de crecimiento y arquitectura radical en plántulas de maíz en condiciones de alta (AP; $1 \mathrm{mM})$ y baja (BP; $1 \mu \mathrm{M})$ disponibilidad de fósforo $\left(\mathrm{KH}_{2} \mathrm{PO}_{4}\right)$.

\begin{tabular}{|c|c|c|c|c|}
\hline \multirow[b]{2}{*}{ Variables } & & \multicolumn{3}{|c|}{ Grupos de eficiencia fósforo } \\
\hline & & $\begin{array}{l}\text { Ineficientes } \\
\text { I (6) }\end{array}$ & $\begin{array}{l}\text { Moderadamente } \\
\text { eficientes } \\
\text { II (53) }\end{array}$ & $\begin{array}{c}\text { Eficientes } \\
\text { III (49) }\end{array}$ \\
\hline \multirow{2}{*}{ Peso seco de raíz (mg/planta) } & $\mathrm{AP}$ & $37.9 \mathrm{~b}$ & $44.7 \mathrm{a}$ & $44.2 \mathrm{a}$ \\
\hline & $\mathrm{BP}$ & $27.9 \mathrm{c}$ & $40.4 \mathrm{~b}$ & $49.2 \mathrm{a}$ \\
\hline \multirow{2}{*}{ Peso seco del vástago (mg/planta) } & $\mathrm{AP}$ & $53.3 \mathrm{~b}$ & $64.2 \mathrm{a}$ & $68.2 \mathrm{a}$ \\
\hline & $\mathrm{BP}$ & $55.4 \mathrm{~b}$ & $65.3 \mathrm{a}$ & $73.6 \mathrm{a}$ \\
\hline \multirow{2}{*}{ Relación raíz: vástago } & $\mathrm{AP}$ & $0.7 \mathrm{a}$ & $0.7 \mathrm{a}$ & $0.6 \mathrm{a}$ \\
\hline & $\mathrm{BP}$ & $0.5 \mathrm{~b}$ & $0.6 \mathrm{a}$ & $0.6 \mathrm{a}$ \\
\hline \multirow{2}{*}{ Longitud de la raíz primaria $(\mathrm{cm})$} & $\mathrm{AP}$ & $27.6 \mathrm{~b}$ & $31.3 \mathrm{a}$ & $32.2 \mathrm{a}$ \\
\hline & $\mathrm{BP}$ & $27.7 \mathrm{~b}$ & $34.1 \mathrm{a}$ & $36.5 \mathrm{a}$ \\
\hline \multirow{2}{*}{ Longitud de la raíz seminal (cm) } & $\mathrm{AP}$ & $24.6 \mathrm{~b}$ & $27.8 \mathrm{a}$ & $28.4 \mathrm{a}$ \\
\hline & $\mathrm{BP}$ & $24.6 \mathrm{~b}$ & $28.1 \mathrm{a}$ & $32.2 \mathrm{a}$ \\
\hline \multirow{2}{*}{ Densidad de pelos radicales en raíz seminal ${ }^{\dagger}$} & $\mathrm{AP}$ & $6.4 \mathrm{a}$ & $6.3 \mathrm{a}$ & $6.2 \mathrm{a}$ \\
\hline & $\mathrm{BP}$ & $7.8 \mathrm{a}$ & $8.1 \mathrm{a}$ & $8.2 \mathrm{a}$ \\
\hline \multirow{2}{*}{ Longitud de pelos radicales en raíz seminal ${ }^{\dagger}$} & $\mathrm{AP}$ & $7.1 \mathrm{a}$ & $6.8 \mathrm{a}$ & $6.8 \mathrm{a}$ \\
\hline & $\mathrm{BP}$ & $8.6 \mathrm{a}$ & $8.5 \mathrm{a}$ & $8.6 \mathrm{a}$ \\
\hline \multirow{2}{*}{ Densidad de pelos radicales en raíz primaria } & $\mathrm{AP}$ & $7.0 \mathrm{~b}$ & $7.9 \mathrm{a}$ & $8.0 \mathrm{a}$ \\
\hline & $\mathrm{BP}$ & $7.5 \mathrm{~b}$ & $8.8 \mathrm{a}$ & $8.8 \mathrm{a}$ \\
\hline \multirow{2}{*}{ Longitud de pelos radicales en raíz primaria } & $\mathrm{AP}$ & $7.5 \mathrm{a}$ & $8.2 \mathrm{a}$ & $8.2 \mathrm{a}$ \\
\hline & $\mathrm{BP}$ & $7.5 \mathrm{~b}$ & $8.8 \mathrm{a}$ & $8.8 \mathrm{a}$ \\
\hline
\end{tabular}

Datos entre paréntesis indican el número de genotipos por grupo. Medias con letras iguales en cada variable son estadísticamente iguales entre sí (Tukey, 0.05).

${ }^{\dagger}$ La densidad y la longitud de los pelos radicales de la raíz primaria y seminal se evaluaron visualmente de acuerdo con una escala de 1 a 9

correlaciones dentro de ambientes se observaron entre longitud de la raíz primaria $(r=0.56 ; r=0.59)$, longitud de la raíz seminal $(r=0.68 ; r=0.69)$, densidad de pelos radicales de la raíz primaria $(\mathrm{r}=0.61 ; \mathrm{r}=0.66)$ y peso seco del vástago $(r=0.51 ; r=0.75)$ en condiciones de AP y BP. De particular relevancia es que ni el peso ni el contenido de fósforo de la semilla de los genotipos eficientes se relacionó con la longitud de la raíz seminal $(r=0.034$; $r=0.026)$, la densidad $(r=0.038 ; r=0.022)$ y longitud de los pelos radicales $(r=0.063 ; r=0.011)$ en condiciones de BP y AP. En los genotipos ineficientes, la longitud de los pelos radicales se relacionó significativamente con el peso de la semilla $(r=0.58 ; r=0.74)$ en condiciones $B P$ y AP, respectivamente.

\section{Asignación de materia seca a la raíz: alometría}

Los patrones de asignación de materia seca a la raíz se relacionaron con los cambios en el crecimiento del vástago y estuvieron afectados por la disponibilidad de fósforo. La correlación entre el logaritmo del peso seco del vástago y el de la raíz a los $7 \mathrm{~d}$ de crecimiento, fue alta en los genotipos ineficientes y moderadamente eficientes en ambos niveles de $\mathrm{P}$ (Figura 3). En los genotipos eficientes la relación fue débil $\left(R^{2}=0.35 ; R^{2}=0.37\right)$ y no significativa, lo que indica que las diferencias observadas en el crecimiento de la raíz no estuvieron determinadas por un efecto alométrico.

\section{DISCUSIÓN}

Los genotipos de maíz de la Meseta P'urhépecha mostraron una amplia variación en su crecimiento con baja disponibilidad de fósforo. El estudio indicó que 49 genotipos de maíz presentaron un mayor crecimiento en $\mathrm{BP}$ y en sus respuestas a la aplicación del tratamiento de AP (Figura 1; Cuadro 2). Cuando se combinaron los valores de eficiencia con la respuesta en crecimiento en alto fósforo, 34 genotipos fueron categorizados como los más eficientes y los de mejor respuesta (Figura 2). Estos genotipos acumularon similares cantidades de materia seca en el vástago, 


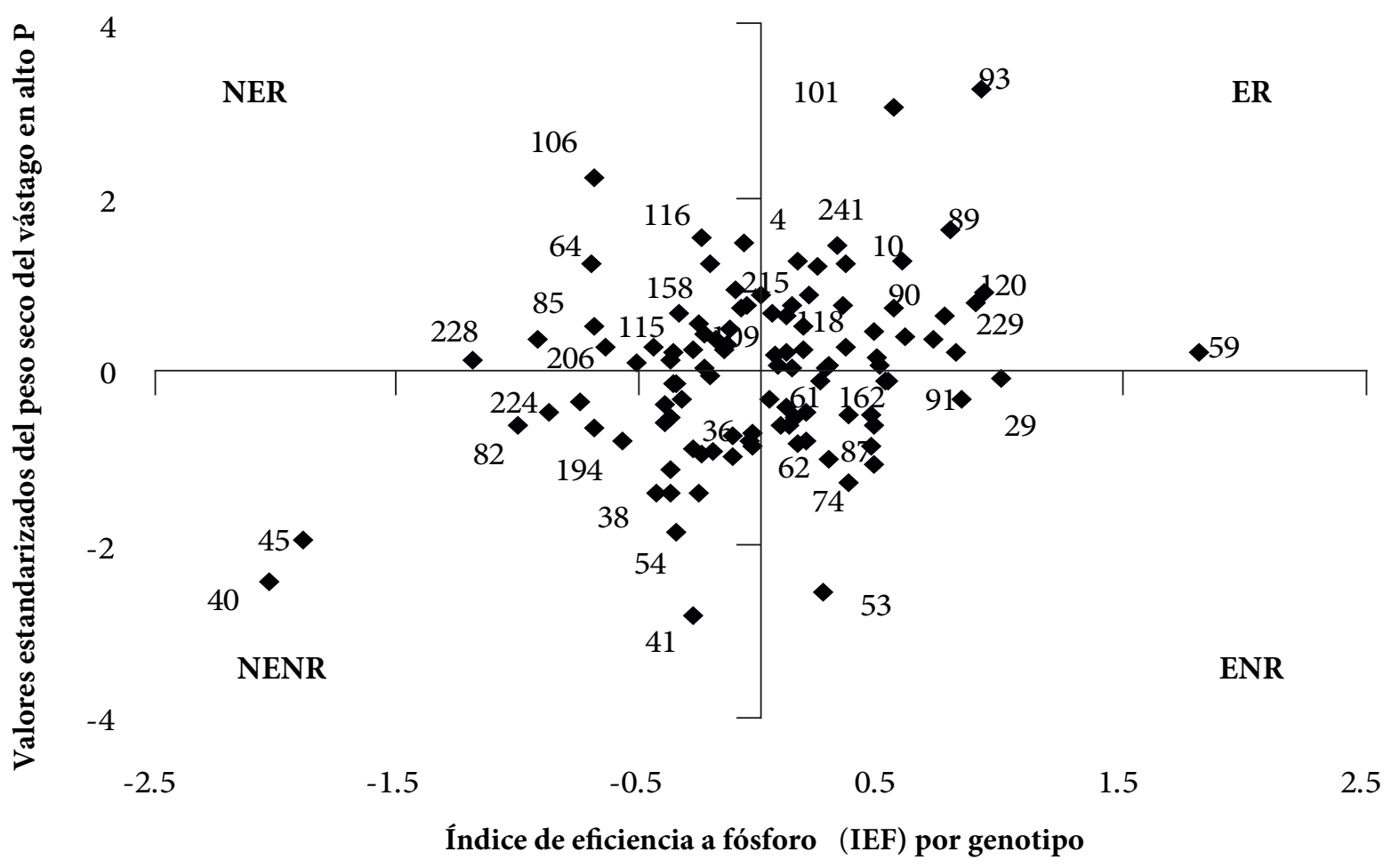

Figura 2. División de 108 genotipos de maíz de acuerdo con el índice de eficiencia a fósforo (IEF) y a los valores estandarizados del peso seco del vástago en condiciones de nivel alto en fósforo. Las categorías de eficiencia y respuesta están representadas por: (i) eficientes y con respuesta (ER), (ii) no eficientes y con respuesta (NER), (iii) no eficientes y sin respuesta e ineficiencia (NENR), y (iv) eficientes y sin respuesta (ENR).

en ambos ambientes (Cuadro 3). Lo anterior evidencia la importancia de identificar germoplasma que sea eficiente en condiciones de deficiencia (ER) y que a la vez tenga un alto potencial de crecimiento en condiciones de alta disponibilidad de fósforo (ENR).

Las diferencias entre los materiales evaluados estuvieron asociadas con la variación en el crecimiento y la arquitectura radical. Los genotipos eficientes presentaron una menor depresión de la materia seca del vástago y un mayor crecimiento de la raíz en condiciones de BP, en comparación con los genotipos ineficientes (Cuadro 2). Según Nielsen et al. (1998), los genotipos eficientes con mayor crecimiento de la raíz pueden adquirir más fósforo, pero este incremento puede repercutir negativamente en el crecimiento de la planta debido a un aumento en la respiración de las raíces. Por su parte, Lynch y Ho (2005) señalaron que los genotipos eficientes de maíz pueden mantener una menor tasa respiratoria (costo de carbono) y mayor crecimiento de la raíz en condiciones limitantes del recurso. En este estudio no se analizaron los costos de asignación de carbono para la producción de raíces, pero se confirmó que los genotipos eficientes mantuvieron una mayor asignación de materia seca a la raíz (Cuadro 2).
La reducida relación alométrica entre el peso seco de la raíz y el vástago de los genotipos eficientes (Figura 3) apoya nuestros hallazgos previos (Bayuelo-Jiménez et al., 2011) en el sentido de que la formación de raíces no está controlada por el tamaño de la planta, sino que depende de la eficiencia en la adquisición de fósforo. Además confirman los reportados por Ochoa et al. (2006) en frijol (P. vulgaris L.) y por Zhu et al. (2006) en maíz. Otro aspecto importante es que el incremento de la longitud de las raíces seminales y la densidad o longitud de los pelos radicales en los genotipos eficientes no estuvo relacionado con el peso de la semilla ni con su contenido de fósforo. Los estudios realizados por Zhu et al. (2004) y Zhu et al. (2005 b) indican que la longitud de la raíz seminal y la formación de pelos radicales pueden incrementar la eficiencia de absorción de $\mathrm{P}$, independientemente del tamaño y reserva de $\mathrm{P}$ en la semilla.

El crecimiento radical y la configuración de las raíces son características clave para optimizar la adquisición y utilización de fósforo en las plantas (Lynch, 2007). La respuesta de las raíces axilares (seminales y nodales) y la longitud de las raíces laterales en condiciones de estrés de $\mathrm{P}$ varía entre genotipos (Zhu y Lynch, 2004). Los genotipos eficientes que 
Cuadro 3. Selección de genotipos de maíz de la Meseta P’urhépecha, Michoacán, con diferente grado de eficiencia (IEF) y respuesta a fósforo (RF), en etapa de plántula.

\begin{tabular}{|c|c|c|c|c|c|c|c|c|}
\hline \multicolumn{2}{|c|}{ Variables } & \multirow{3}{*}{$\begin{array}{c}\text { Madurez } \\
\text { Precoz }\end{array}$} & \multirow{3}{*}{$\begin{array}{c}\mathrm{GM}^{\dagger} \\
\mathrm{C}\end{array}$} & \multicolumn{5}{|c|}{$\begin{array}{c}\text { Peso seco de vástago } \\
\text { (mg/planta) }\end{array}$} \\
\hline & & & & \multirow{2}{*}{$\begin{array}{c}\mathrm{BP} \\
72.5\end{array}$} & \multirow{2}{*}{$\frac{\mathrm{AP}}{70.0}$} & \multirow{2}{*}{$\frac{\text { IEF }}{0.18}$} & \multirow{2}{*}{$\frac{\text { Sd_PSV }^{\dagger \dagger}}{0.25}$} & \multirow{2}{*}{$\frac{\mathrm{RF}^{+\dagger \dagger}}{\mathrm{ER}}$} \\
\hline 103 & UR-9 & & & & & & & \\
\hline 233 & Macho-I-03 & Precoz & S & 87.5 & 80.0 & 0.24 & 1.20 & ER \\
\hline 10 & ZPT-6 & Precoz & $\mathrm{C}$ & 87.5 & 82.5 & 0.59 & 1.26 & ER \\
\hline 93 & ZPT-11 & Precoz & $\mathrm{C}$ & 75.0 & 107.5 & 0.91 & 3.25 & ER \\
\hline 101 & UR-3 & Precoz & $\mathrm{C}$ & 75.0 & 105.0 & 0.56 & 3.04 & ER \\
\hline 89 & PICH-18 & Intermedio & $\mathrm{C}$ & 95.2 & 87.5 & 0.79 & 1.64 & ER \\
\hline 154 & San Juan Tumbio 2 & Intermedio & $\mathrm{CM}$ & 75.0 & 70.0 & 0.81 & 0.21 & ER \\
\hline 205 & Turirán & Intermedio & $\mathrm{HxC}$ & 82.5 & 75.0 & 0.01 & 0.86 & ER \\
\hline 215 & Zacapu & Intermedio & $\mathrm{CM}$ & 85.0 & 75.0 & 0.21 & 0.86 & ER \\
\hline 4 & SANAZ-5 & Tardío & $\mathrm{C}$ & 73.3 & 85.0 & 0.32 & 1.45 & ER \\
\hline 229 & Turirán amarillo & Tardío & $\mathrm{CM}$ & 85.0 & 75.0 & 0.77 & 0.63 & ER \\
\hline 231 & Macho-III-04 & Tardío & S & 92.5 & 77.5 & 0.89 & 0.78 & ER \\
\hline 96 & ZPT-4 & Precoz & $\mathrm{C}$ & 65.0 & 85.0 & -0.07 & 1.47 & NER \\
\hline 99 & TZU-7 & Precoz & $\mathrm{C}$ & 77.5 & 80.0 & -0.20 & 1.24 & NER \\
\hline 85 & PICH-17 & Precoz & $\mathrm{C}$ & 52.5 & 72.5 & -0.68 & 0.52 & NER \\
\hline 106 & UR-6 & Precoz & $\mathrm{C}$ & 77.5 & 95.0 & -0.68 & 2.23 & NER \\
\hline 123 & Turirán CM-00-05 & Intermedio & $\mathrm{CM}$ & 55.0 & 77.5 & -0.06 & 0.77 & NER \\
\hline 64 & PICH-M-19 & Intermedio & $\mathrm{C}$ & 60.0 & 82.5 & -0.70 & 1.22 & NER \\
\hline 182 & Macho-III-05 & Intermedio & S & 67.5 & 72.5 & -0.12 & 0.48 & NER \\
\hline 208 & Bonilla & Intermedio & $\mathrm{HxC}$ & 70.0 & 77.5 & -0.10 & 0.94 & NER \\
\hline 140 & Cruz Gorda CM-00-05 & Precoz & $\mathrm{CM}$ & 52.5 & 57.5 & -0.68 & -0.66 & NENR \\
\hline 40 & PA-JRG-13-Mz 4 & Intermedio & $\mathrm{C}$ & 37.5 & 32.5 & -2.02 & -2.44 & NENR \\
\hline 41 & PM-TA-16-Mz 21 & Tardío & $\mathrm{C}$ & 47.5 & 27.5 & -0.27 & -2.84 & NENR \\
\hline 111 & Coangatzio CM-00-05 & Precoz & $\mathrm{CM}$ & 70.0 & 50.0 & 0.47 & -1.09 & ENR \\
\hline 91 & SANAZ & Precoz & $\mathrm{C}$ & 100.0 & 62.5 & 0.52 & -0.13 & ENR \\
\hline 74 & OP-5 & Intermedio & $\mathrm{C}$ & 62.5 & 50.0 & 0.29 & -1.02 & ENR \\
\hline 29 & UR-10 & Intermedio & $\mathrm{C}$ & 87.5 & 60.0 & 0.83 & -0.34 & ENR \\
\hline 62 & PICH-1 & Intermedio & $\mathrm{C}$ & 72.5 & 55.0 & 0.19 & -0.81 & ENR \\
\hline 9 & NURO-1 & Tardío & $\mathrm{C}$ & 70.0 & 57.5 & 0.08 & -0.64 & ENR \\
\hline 57 & CB-9 & Tardío & $\mathrm{C}$ & 70.0 & 57.5 & 0.13 & -0.62 & ENR \\
\hline 61 & OP-1 & Tardío & $\mathrm{C}$ & 72.5 & 57.5 & 0.20 & -0.49 & ENR \\
\hline 185 & CIMMYT-1 M-00-05 & Tardío & $\mathrm{HxC}$ & 72.5 & 57.5 & 0.47 & -0.51 & ENR \\
\hline \multirow[t]{3}{*}{189} & El Tigre CM-00-05 & Tardío & $\mathrm{CM}$ & 69.5 & 52.5 & 0.15 & -0.83 & ENR \\
\hline & Media general & & & 68.5 & 65.4 & & & \\
\hline & DMS (0.05) & & & 13.1 & 12.1 & & & \\
\hline
\end{tabular}

${ }^{\dagger}$ Grupo de mejoramiento: C, Criollo; CM, Criollo mejorado; HxC, híbrido x criollo y S, híbrido mejorado.

ॠValores estandarizados del peso seco del vástago (Sd_PSV) en condiciones de AP.

${ }^{++}$Respuesta a fósforo (RF) expresada como peso seco del vástago en AP: (i) eficientes y con respuesta (ER), (ii) no eficientes y con respuesta (NER), (iii) no eficientes y sin respuesta e ineficiencia (NENR), y (iv) eficientes y sin respuesta (ENR).

${ }^{*}$ Diferencia mínima significativa para comparar entre medias de genotipos. 


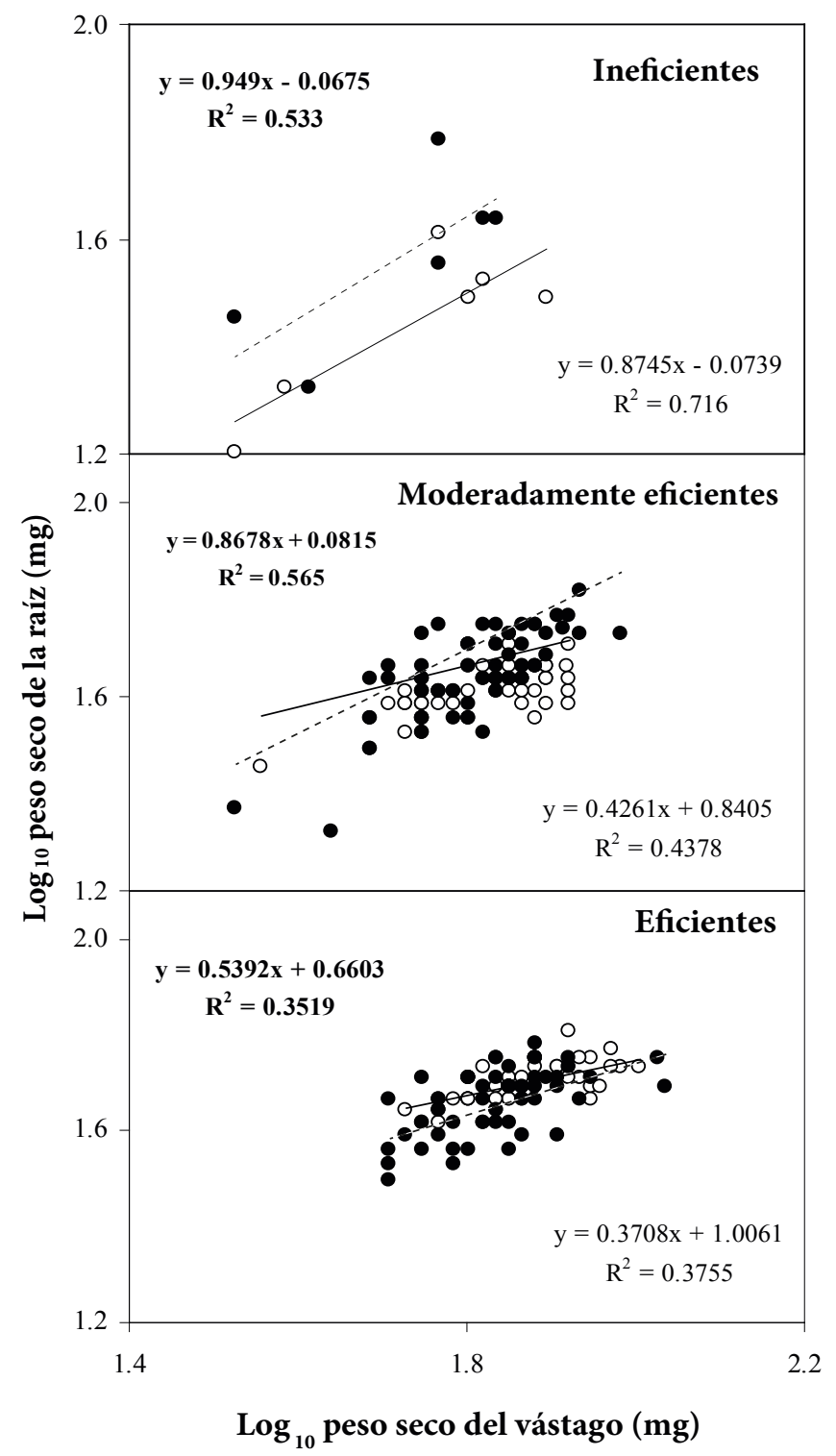

Figura 3. Relación alométrica entre el peso seco de la raíz $\left(\log _{10} \mathrm{mg}\right)$ y el peso seco del vástago $\left(\log _{10} \mathrm{mg}\right)$ de 6, 53 y 49 genotipos de maíz ineficientes, moderadamente eficientes y eficientes a fósforo desarrollados en nivel alto $(\bigcirc)$ y bajo $(\bigcirc)$ en fósforo.

incrementan las raíces axilares bajo deficiencia de fósforo pueden mantener un crecimiento continuo en la planta y son más eficientes para adquirirlo en la superficie del suelo (Miller et al., 2003; Zhu et al., 2005b; Ochoa et al., 2006).

En etapa de plántula la mayor parte del sistema radical de maíz lo constituyen las raíces seminales, las cuales contribuyen eficazmente a aumentar la adquisición de $\mathrm{P}$ en condiciones restrictivas (Zhu et al., 2006). En este estudio se confirmó que el incremento de la longitud de las raíces seminales estuvo correlacionado con la eficiencia a fósfo- ro $(r=0.68)$. Los genotipos eficientes con raíces seminales más largas acumularon más materia seca en el vástago que los genotipos ineficientes, en condiciones de BP. Estudios realizados con QTLs (loci de rasgos cuantitativos, por sus siglas en inglés) han demostrado que existe variación genética en las raíces seminales de maíz en etapa de plántula, y que dicho carácter está regulado por genes que se expresan en condiciones de reducida disponibilidad (Zhu et al., 2006).

Los pelos radicales son proyecciones tubulares de las 
células epidérmicas y contribuyen con la adquisición de $\mathrm{P}$ (ion con bajo coeficiente de difusión) en el suelo (Jungk, 2001). En condiciones limitantes de fósforo es común que incremente la longitud de los pelos radicales (Bates y Lynch, 2001) y su densidad (Ma et al., 2001). Los resultados de este estudio corroboraron que la deficiencia de fósforo estimuló el incremento en la densidad y longitud de pelos radicales en la raíz primaria y seminal de los genotipos eficientes (Cuadro 2). La presencia de pelos más densos en la raíz seminal y primaria se asoció con un mejor crecimiento en condiciones de BP ( $r=0.56$ a 0.66$)$, como otros investigadores han reportado en plántulas de maíz (Zhu et al., 2005a). Estos últimos autores sugieren que la variación genética en la densidad de los pelos radicales está controlada por siete QTLs y que estas características podrían ser de utilidad en programas de mejoramiento asistido (Ochoa y Lynch, 2006). Los resultados de este estudio sugieren que las características radicales de maíz son un factor determinante en la adquisición de fósforo y en la expresión de su eficiencia, en etapa de plántula y en posteriores estados del desarrollo (Bayuelo-Jiménez et al., 2011).

\section{CONCLUSIÓN}

Los genotipos de maíz de la Meseta P'urhépecha mostraron gran variabilidad en el crecimiento vegetal, en la eficiencia a fósforo y en la arquitectura radical bajo condiciones de limitada disponibilidad de fósforo. La eficiencia a fósforo estuvo determinada por una mayor producción de materia seca en la raíz y el vástago, así como una mayor asignación de materia seca a la raíz, raíces seminales de mayor longitud y pelos radicales más densos en la raíz seminal y primaria. La variación de estos caracteres podría ser de utilidad en programas de mejoramiento vegetal enfocado a incrementar la eficiencia en la adquisición de fósforo.

\section{AGRADECIMIENTOS}

Al Organismo Internacional de Energía Atómica en Viena, Austria (Proyecto RO-13800) y a la Coordinación de Investigación Científica (Proyecto 6.11), Universidad Michoacana de San Nicolás de Hidalgo, por el apoyo financiero otrogado.

\section{BIBLIOGRAFÍA}

Alcalá de Jesús M, C A Ortíz-Solorio, M C Gutiérrez-Castorena (2001) Clasificación de los suelos de la Meseta Tarasca, Michoacán. Terra 19:217-239.

Bayuelo-Jiménez J S, V A Pérez-Decelis, M L Magdaleno-Armas, M Gallardo-Valdéz, I Ochoa, J P Lynch (2011) Genetic variation for root traits of maize (Zea mays L.) from Purhepecha Plateau, under contrasting phosphorus availability. Field Crops Res. 121:350-362.

Bates T, J P Lynch (2001) Root hairs confer a competitive advantage under low phosphorus availability. Plant Soil 236:243-250.

Bonser A M, J P Lynch, S Snapp (1996) Effect of phosphorus deficiency on growth angle of basal roots of Phaseolus vulgaris L. New
Phytol. 132:281-288.

Fageria N K, V C Baligar, Y C Li (2008) The role of nutrient efficient plants in improving crop yields in the twenty first century. J. Plant Nutr. 31:1121-1157.

Hinsinger $P$ (2001) Bioavailability of soil inorganic $P$ in the rhizosphere as affected by root-induced chemical changes: a review. Plant Soil 237:173-195

Hochholdinger F, K Woll, M Saues, D Dembinsky (2004) Genetic dissection of root formation in maize (Zea mays) reveals roottype specific development programmers. Ann. Bot. 93:359368.

Jungk A (2001) Root hairs and the acquisition of plant nutrients from soil. J. Plant Nutr. Soil Sci. 162:9-24.

Kochian L V, M A Pineros, O A Hoekenga (2005) The physiology, genetics and molecular biology of plant aluminium resistance and toxicity. Plant Soil 264:175-195.

Li S C, J Gong (2003) Screening maize inbred lines seedling for tolerance to low phosphate stress. J. Maize Sci. 11:85-89.

Lynch J P (2007) Roots of the second Green Revolution. Aust. J. Bot 55:493-512.

Lynch J P, M D Ho (2005) Rhizoeconomics: Carbon costs of phosphorus acquisition. Plant Soil 268:45-56.

Ma Z, T C Walk, A Marcus, J P Lynch (2001) Morphological synergism in root hair length, density, initiation and geometry for phosphorus acquisition in Arabidopsis thaliana: a modelling approach. Plant Soil 236:221-235.

Miller C, I Ochoa, K L Nielsen, D Beck, J P Lynch (2003) Genetic variation for adventitious rooting in response to low phosphorus availability: potential utility for phosphorus acquisition from stratified soils. Funct. Plant Biol. 30:973-985.

Murphy J, J P Riley (1962) A modified single solution method for the determination of phosphate in nature waters. Anal. Chim. Acta 27:31-36.

Nielsen K L, T J Bouma, J P Lynch, D M Eissenstat (1998) Effects of phosphorus availability and vesicular-arbuscular mycorrhizas on the carbon budget of common bean (Phaseolus vulgaris L.). New Physiol. 139:647-656.

Newton A C, T Akar, J P Baresel, P Bebeli, E Bettencourt, K V Bladenopoulus, J H Czembor, D A Fasoula, A Katsiotis, $K$ Koutis, M Koutsika, G Kovacs, H Larsson, M A Pinheiro de Carvalho, D Rubiales, J Russel, T M Dos Santos, M C Vaz Patto (2010) Cereal landraces for sustainable agriculture. A review. Agron. Sust. Develop. 30:237-269.

Ochoa I, M W Blair, J P Lynch (2006) QTL Analysis of adventitious root formation in common bean (Phaseolus vulgaris $\mathrm{L}$.) under contrasting phosphorus availability. Crop Sci. 46:1609-1621.

Pan X W, W B Li, Q Y Zhang, Y H Li, M S Liu (2008) Assessment on phosphorus efficiency characteristics of soybean genotypes in phosphorus-deficient soils. Agric. Sci. China 7:958-969.

Richardson E, J Lynch, P R Ryan, E Dehaize, FF A Smith, S E Smith, P R Harvey, M H Ryan (2011) Plant and microbial strategies to improve the phosphorus efficiency in agriculture. Plant Soil 349:121-156

Romerburg H C (1984) Cluster Analysis for Researches. Lifetime Learning Publications Belmont, California. $277 \mathrm{p}$.

SAS Institute (2000) SAS User's Guide: Statistics. SAS Institute, Inc., Cary, NC.

Vance C P, C Udhe-Stone, D L Allan (2003) Phosphorus acquisition and use: critical adaptation by plants for securing a nonrenewable resource. New Phytol. 197:423-447.

Vieira R F, C N Jochua, J P Lynch (2007) Method for evaluation of root hairs of common bean genotypes. Pesq. Agropec. Bras. 42:1365-1368.

Zhu J, J P Lynch (2004) The contribution of lateral rooting to phosphorus acquisition efficiency in maize (Zea mays L.) seedlings. Funct. Plant Biol. 31:949-958.

Zhu J, S M Kaeppler, J P Lynch (2005a) Mapping of QTL controlling root hair length in maize (Zea mays L.) under phosphorus deficiency. Plant Soil 270: 299-310.

Zhu J, S M Kaeppler, J P Lynch (2005b) Mapping of QTL for lateral root branching and length in maize (Zea mays L.) under differential phosphorus supply. Theor. Appl. Genet. 111:688-695. 
Zhu J, S M Mickelson, S M Kaepler, J P Lynch (2006) Detection of quantitative trait loci for seminal root traits in maize (Zea mays L.) seedlings grown under differential phosphorus levels. Theor. Appl. Genet. 113:1-10. 